\title{
Metronidazole resistance in anaerobes isolated from patient with oral and maxillofacial infections attending muhimbili national hospital, dar-es-salaam, Tanzania
}

\begin{abstract}
Background: Oro-facial infections, mostly bacterial infections, are among the most commonly encountered problems in the oral and maxillofacial surgical practice world over. These infections if not properly diagnosed and treated usually result in significant morbidity and are often fatal. The infection is caused by both aerobic and anaerobic microorganism where as treatment is given using broad spectrum antibiotics mostly common combination of Amoxicillin and Metronidazole. Metronidazole is commonly targeted drug for anaerobes, however recently the anaerobes have shown resistance to this drug. The aim of this study was to isolate and identify anaerobes associated with oral and maxillofacial infection and their susceptibility pattern to Metronidazole in patients attending Muhimbili National Hospital, Dar-es-salaam, Tanzania.
\end{abstract}

Method: Seventy (70) samples were collected from patients with various forms of oral facial and infections that consented and were recruited for the study. The samples were processed for isolation and identification of bacteria as per standard microbiological techniques. The antimicrobial susceptibility was done by E-test (Biomerieux) methods as per CLSI guidelines.

Results: Anaerobes were isolated in $83 \%$ of oral and maxillofacial cases. Anaerobes were found to be over $99 \%$ sensitive to metronidazole while some isolated showed approximately $40 \%$ resistance to Metronidazole. Majority of isolated anaerobes display good sensitivity.

Conclusion: Resistance to Metronidazole observed in this study and also presence of anaerobes in these patients showed that there is a need to isolate anaerobes especially in patient with severe oral and maxillofacial infections and study their antimicrobial susceptibility pattern for better management.

Keywords: anaerobes, oral and maxillofacial infections, metronidazole
Volume 5 Issue 2 - 2017

\author{
Sima E Rugarabamu \\ Department of Microbiology and Immunology, Muhimbili \\ University of Health and Allied Sciences, Tanzania
}

Correspondence: Sima E Rugarabamu, Department of Microbiology and Immunology, Muhimbili University of Health and Allied Sciences, Tanzania, Email sima_luv@yahoo.com

Received: May 26, 2017 | Published: June 22, 2017

\section{Background}

Oro-facial infections, mostly bacterial infections, are among the most commonly encountered problems in the oral and maxillofacial surgical practice world over. Infections involving the oral and maxillofacial region usually result in significant morbidity and are often fatal. These infections. ${ }^{1}$ Purulent infections such as dental abscesses, Ludwing Angina are known to be caused by not only aerobes and facultative anaerobes but also by obligate anaerobes. ${ }^{2}$ An increase in the role of these bacteria in clinical infections has been reported, however, exact incidence especially to anaerobes is unknown. ${ }^{3}$ In Many Oral and maxillofacial clinics patients present with serious and often fatal infections that initially started as simple dental caries or periodontitis then progressed to periapical infection and spread to facial spaces/ In severe forms of orofacial infections like necrotizing fasciitis, deep space infection and osteomyelitis, culture studies involving both aerobic and anaerobic bacteriology are desirable to provide information on the likely pathogenic organisms causing the disease and their likely antibiotic sensitivity. ${ }^{4}$ This is not done because isolation and sensitivity testing of anaerobes is very tedious, costly and time consuming. As a result treatment is given using broad spectrum antibiotics which may not be appropriate or may cause delay in healing and high morbidity.

Detection of bacteria along with their susceptibility pattern is important for better management of infections since some organism are now developing resistance to commonly used antimicrobial for infections..$^{5}$ Anaerobic bacteria are predominantly implicated in the pathogenesis of oral and maxillofacial infections hence their detection is important for better management of anaerobic infections. However, most laboratories do not attempt to isolate or do the susceptibility testing of anaerobes as it requires special expertise and expensive facilities. ${ }^{6}$ Metronidazole is commonly targeted drug for anaerobes, however recently the anaerobes have shown resistance to this drug. It was therefore decided to study isolate and identify anaerobes associated with oral and maxillofacial infection and their susceptibility pattern to Metronidazole in patients attending Muhimbili National Hospital, Dar-es-salaam, Tanzania.

\section{Material and methods}

This was a descriptive cross-sectional hospital based study that took place between January 2014 to July 2014 at Muhimbili National Hospital (MNH) which is the largest referral, consulting and teaching hospital in Tanzania and was divided into two parts. This was conducted in the department of oral and maxillofacial surgery clinic in the school of dentistry. The clinic attends referral and non-referral patients from Dar-es-salaam and neighboring district and up country clinics. This was done in microbiology laboratory of Muhimbili University Dar -Es-Salaam. 


\section{Study population}

All patients who attended treatment and were diagnosed to have oral and maxillofacial infections at the Oral and Maxillofacial OPD and those admitted in oral and maxillofacial wards of $\mathrm{MNH}$ were eligible for the study. Enrollment was subject to obtaining an informed verbal consent.

\section{Data collection}

Interview was conducted was conducted using a structured standard questionnaire to obtain information regarding age, sex, the presenting symptoms, duration of the condition and medical history.

\section{Clinical examination}

General physical examination was carried out followed by extra oral and intraoral examination with the patient seated on a dental chair using natural or artificial light at the OPD. For Admitted patient's examination was carried out while lying on an examination bed. A special clinical form was used to record the following; Source of infection characteristic of the lesion, location, pain and tenderness, discharge and foul smell.

\section{Isolation and identification of organism}

Sample collection and transportation: Pus samples were collected aseptically by aspirating the abscesses using sterile syringe during the incision and drainage or wound dressing. After aspirating the specimen was immediately inoculated in two media, one special anaerobic transport media (BD curls anaerobic Transport) and the other universal container. Specimen was transferred to the laboratory within $20 \mathrm{~min}$ processed for culture as early as possible within $2 \mathrm{hrs}^{7}{ }^{7}$ The culture and sensitivity were conducted for the clinical specimens obtained from the patient before initiation of any antibiotic therapy. Sample not suitable for culture such as contaminated or those that did not meet the criteria such as exposure to antibiotic was discarded.

\section{Specimen processing}

Direct smear: This formed a crucial role in the processing of specimen by giving preliminary diagnosis of infection. Gram stain was done followed by examination under a microscope using oil immersion $(100 \times$ magnification), pus cells, bacteria cells and other characteristics such as fine slender, minute, pleomorphic features were appreciated. Samples from universal container were processed for aerobic culture to look for growth of aerobes and facultative anaerobes. After gram staining samples were inoculated on to $(5 \%)$, sheep blood agar, chocolate agar and Mac Conkey agar. The plates were incubated aerobically at 370C (Mac Conkey) and under 5\% $\mathrm{CO}_{2}$ (Blood and Chocolate) and examined after 24h. After incubation predominant colonies were processed further and identified conventional methods. For anaerobic culture Blood agar containing kanamycin and Vancomycin (BD0403 CDC 5\% sheep blood agar for anaerobes) was used. $5 \mu \mathrm{g}$ metronidazole and $10 \mu \mathrm{g}$ penicillin discs and $10 \mu \mathrm{g}$ Gentamycin was placed for presumptive recognition of anaerobes and the media was incubated in anaerobic jar in atmosphere generated using $\mathrm{BD}$ commercial gas generating kit in accordance with manufacturer's instructions. Anaerobic plates were examined after $48 \mathrm{~h}$ Isolates were identified based on microscopic characteristics, colonial characteristic and biochemical test. ${ }^{5}$

\section{Antimicrobial susceptibility testing}

Antimicrobial susceptibility pattern of isolated aerobic bacterial pathogens was conducted by using $\mathrm{E}$ test (Biomerieux) anaerobic bacteria were determined by E test (Biomerieux, Sweden) according to CLSI guideline for anaerobic susceptibility testing.

\section{Results}

\section{Isolated anaerobes}

A total of $58(83 \%)$ anaerobic organisms were isolated with the most common isolate being Prevotella 25(43\%) followed by Peptostreptococcus 18(31\%) Other isolates were, Pophyromonas spp. 3(5\%), Fusobacterium spp. 2(3\%) and Bacteroides spp. 5(9\%) (Table $1 \& 2$ ).

Table I Isolation frequency of obligatory anaerobic organisms from oral facial infection

\begin{tabular}{lll}
\hline Bacteria no of isolate (\%) & & \\
\hline Prevotella spp & 25 & 43 \\
Peptostreptococus & 18 & 31 \\
Bacteroides & 5 & 9 \\
Fusobacteria & 2 & 3 \\
Actinomyces & 1 & 2 \\
Pophyromonas & 3 & 5 \\
Non Identified Anaerobes & 4 & 7 \\
Total & 58 & 100 \\
\hline
\end{tabular}

Table 2 Sensitivity pattern of anaerobes isolated from oral facial infection

\begin{tabular}{lllllll}
\hline \multirow{2}{*}{ Isolate } & \multicolumn{6}{l}{ Susceptibility (\%) } \\
\cline { 2 - 7 } & Pen & Cefo & Mtr & Pen & Chlo & penem \\
\hline Prevotella & 65 & 95 & 60 & 90 & 95 & 95 \\
P.streptococcus & 88 & 94 & 88 & 94 & NT & NT \\
Bacteroides & 80 & 100 & 60 & 80 & 100 & 80 \\
Pophyromonas & 100 & 100 & 67 & 100 & 100 & 100 \\
Fusobacteria & 100 & 100 & 50 & 100 & 100 & 100 \\
\hline
\end{tabular}

Mtr: Metronidazole; Chlo: Chloramphenicol; Tet: Tetracycline; Pen: Penicillin; Cefo:Am/Clavum= Clavulanic acid Amoxicilim; penem: Ibipenem.

\section{Discussion}

In this study a total of 70 samples were collected from different clinical conditions such as Dental abscesses, Ludwing's angina, Necrotising fasciitis and infected socket. Anaerobes were isolated in $83 \%$ which is in agreement with other studies that reported that infection due to anaerobes have increased in comparison to past reports ${ }^{8}$ and also in this study the superior methods of collection of specimens and culture was applied hence could have attributed to improving their isolation. Regarding the bacterial species that were isolated, the findings of this study are in agreement with other researchers who also demonstrated that Streptoccus species, Peptostreptococus, Prevotella/ Bacteroides, Fusobacteria and others as frequently isolated organisms from oral and maxillofacial infections. ${ }^{8-12}$ Metronidazole which is commonly used had a questionable activity in regarding to the similar to other studies that has recently reported resistance to anaerobes isolated from head and neck and other parts of the body.

\section{Conclusion}

Metronidazole is among the commonest drug used to treat oral facial and maxillofacial infections. However no much data is available on its sensitivity in anaerobes isolated in this region. Results of this study should therefore be an highlight on the need to isolate and study the antimicrobial susceptibility pattern of various drugs used to treat patients with oral and maxillofacial infections for better management of patients. 


\section{Acknowledgements}

I express my deep gratitude for the cooperation extended by the patients who were involved in the study and appreciate assistance received from all the worker in the Microbiology Lab.

\section{Conflicts of interest}

The authors declare that there is no conflict of interest.

\section{References}

1. Robertson D, Smith AJ. The microbiology of the acute dental abscess. $J$ Med Microbiol. 2009;58(2):155-162.

2. Tanner N Stillman. Oral and Dental Infections with Anaerobic-Bacteria - Clinical-Features, Predominant Pathogens, and Treatment. Clin Infect Dis. 1993;16(4):S304-S309.

3. Simon E, Matee MI. Cervico-facial necrotising fasciitis occurring with facial paralysis: Case report. East Afr Med J. 1999;76(8):472-474.

4. Akinkunmi EO, Adesunkanmi AR, Lamikanra A. Pattern of pathogens from surgical wound infections in a Nigerian hospital and their antimicrobial susceptibility profiles. Afr Health Sci. 2014;14(4):802809.

5. Manyahi J. Bacteriological spectrum of post-operative wound infections and their antibiogram in a tertiary hospital, Dar es Salaam, Tanzania. Muhimbili University of Health and Allied Sciences; 2012:1-78.
6. Brook EH Frazier, Gher ME. Microbiology of periapical abscesses and associated maxillary sinusitis. J Periodontol. 1996;67(6):608-610.

7. Brook. The role of beta-lactamase-producing-bacteria in mixed infections. BMC Infect Dis. 2009;9:202.

8. Catherine AS, Myrana TM, Marrisa MA, et al. Microbiologic and clinical profile of anaerobic diabetic foot infections. Phil J Microbiol Infect Dis. 2002;31(4):151-160.

9. Pednekar SN, Pol SS, Agrawal SA, et al. Metronidazole resistance in anaerobes isolated from chronic periodontitis cases. $J$ Evolution Med Dent Sci. 2016;5(4):270-271.

10. Michaela Stieglmeier, Reinhard Wirth, Gerhard Kminek, et al. Cultivation of Anaerobic and Facultatively Anaerobic Bacteria from Spacecraft-Associated Clean Rooms. Appl Environ Microbiol. 2009;75(11):3484-3491.

11. Akhi MT, Ghotaslou R, Beheshtirouy S, et al. Antibiotic Susceptibility Pattern of Aerobic and Anaerobic Bacteria Isolated From Surgical Site Infection of Hospitalized Patients, Jundishapur. $J$ Microbiol. 2015;8(6):e20309.

12. Holmstrup P, Poulsen AH, Andersen L, et al. Oral infections and systemic diseases. Dent Clin North Am. 2003;47(3):575-598. 